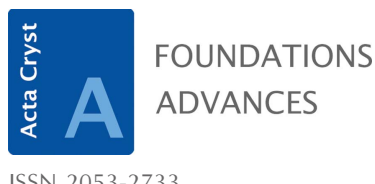

ISSN 2053-2733

\title{
Shattered Symmetry. Group Theory From the Eightfold Way to the Periodic Table. By Pieter Thyssen and Arnout Ceulemans. Oxford University Press, 2017. Pp. 528. Price GBP 52.00 (hardback). ISBN 9780190611392.
}

\author{
Xavier Assfeld*
}

Université de Lorraine, CNRS, SRSMC, Nancy, France. *Correspondence e-mail: xavier.assfeld@univ-lorraine.fr

Keywords: book review; group theory; Lie groups.

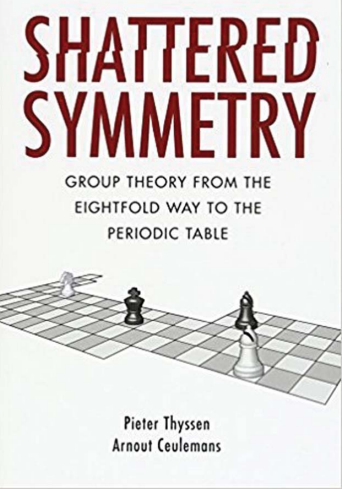

(C) 2018 International Union of Crystallography
The subtitle of this marvellous book could have been: 'All you always wanted to know about Lie groups, but were too afraid to ask'. Most of the time, books dealing with such a subject propose a mathematical treatment that goes well beyond the basic calculus knowledge of physicists and chemists. This one uses simple calculus and takes the reader by the hand through all chapters in a step-by-step way; difficulties are handled gradually in a very pedagogical presentation. It starts with the example of usual groups - usual in the sense that they are known by every chemist or physicist - and ends with the proposition of a very complex Lie group $[\mathrm{SO}(4,2)]$ to explain, or at least to rationalize, the structure of the periodic table and the $(n+l)$ rule. Every chapter provides the reader with a snippet of new knowledge, in order to allow her or him to appreciate and understand each landscape at the heart of the Lie-group world. The reader will also find a collection of historical anecdotes and illustrations based on the journey of Alice when she goes through the mirror, just as the reader travels across the wonderland of Lie groups and their implications in physics and chemistry. As may be apparent, I really appreciated reading this book and I recommend it to anyone willing to understand this powerful tool. Below, this review comments on each of the 14 chapters and the 12 appendices in order of appearance.

The first chapter is dedicated to the definition of several notions needed to grasp the meaning of the remaining parts of the book. It is based on the point group of the triangle that everyone can understand. The inspiring life of Evarist Gallois is detailed to give historical markers.

With the second chapter one takes a step through the mirror with the elements of group theory and the concept of symmetry breaking, isomorphisms and homomorphisms. Again, usual groups are used to guide the reader and an historical interlude concludes the chapter.

Armed with definitions from the first two chapters, the reader can take a step further into the world of continuous groups with the study of the axial rotation group which is the subject of the third chapter. The matrix representations are discovered and used to build the $\mathrm{O}(2)$ and $\mathrm{SO}(2)$ groups.

The latter is used throughout the fourth chapter to introduce the infinitesimal generators that are going to be used in the following chapters. The $\pi$ system of planar ring molecules, which is well known by chemists, is used to illustrate the power of group theory and in particular the group $\mathrm{SO}(2)$.

The fifth chapter adds some complexity to the study in going from rotation in a plane to rotation in three dimensions. The $\mathrm{SO}(3)$ and $\mathrm{O}(3)$ groups are built and most importantly the Lie algebra is presented together with Casimir invariants. The role of these invariants in quantum mechanics is tremendous. In a similar way to the previous chapter, the fifth one ends with the study of the $\pi$ system of buckminsterfullerene.

All features presented in the first five chapters are mathematically grounded in the scholium that forms Chapter 6. Here the three steps connecting the Lie algebra of a Lie group to the spectrum of the Hamiltonian are detailed going from the Cartan subalgebra and Cartan generators to Casimir invariants via Weyl generators and Weyl diagrams. It is certainly the most important chapter and it ends the first part of the book, focused on space symmetries. 
The second part is concerned with dynamic symmetries and starts with the seventh chapter, which deals with the SU(3) [and $\mathrm{U}(3)$ ] group. The whole jungle of elementary particles is rationalized with this group and the reader can appreciate the concept of symmetry breaking. Harmonic oscillators in one and three dimensions are used to illustrate the use of operators. As may be noticed, complexity is increasing from the $\mathrm{O}(2)$ group to $\mathrm{SU}(3)$ via $\mathrm{SO}(3)$. It decreases a little bit in the next chapter.

SU(2) applied to the electron spin is the main subject of Chapter 8. If Chapter 7 was quite long (65 pp.), Chapter 8 is quite short at $8 \mathrm{pp}$. This chapter is much less attractive than the previous ones. It feels as if something is missing. The chapter dives straight into the theory without any warning or explanation. Even though the chapter is technically sound, it deserves the same charming style that the other chapters have.

Chapter 9 discusses the hydrogen atom, the angular stone of quantum chemistry. It is studied with the help of the $\mathrm{SO}(4)$ group and, even if it is an old story, there is still something to learn from the point of view of group theory.

The second scholium constitutes Chapter 10 and ends the second part of the book. The hydrogen atom is revisited as a four-dimensional harmonic oscillator taking full advantage of the $\mathrm{SO}(4)$ group structure.
The last part of the book, Part 3, is about spectrum-generating symmetries. The time variable is introduced and the $\mathrm{SO}(n, l)$ groups are defined. $\mathrm{SO}(2,1)$ is the first group of this kind. Its relation with $\mathrm{SO}(3)$ is examined and it is used to define creation and annihilation operators.

Chapter 12 deals with a much more complex group, namely $\mathrm{SO}(4,2)$ where four dimensions of space and two of time are considered. The same methodology, see Chapter 6, is applied to decipher all the invariants.

The periodic table is the main subject of Chapter 13. Its structure and the governing laws of atomic levels are reviewed. Its symmetry properties are described and the reader is reminded that there are as yet no theoretical arguments to explain the $(n+l)$ rule. Chapter 14 proposes to rationalize the structure of the periodic table with the help of the $\mathrm{SO}(4,2)$ group in the same way as the zoo of elementary particles was put in order with the $\mathrm{SO}(3)$ group. It is amusing that the various operators are interpreted as chess pieces.

Finally, 12 appendices conclude this book containing all the necessary mathematical demonstrations. Their inclusion in the text would have made reading cumbersome.

As already stated, this book is a perfect guide for physicists and chemists willing to learn about Lie groups and their applications to physical or chemical problems. I heartily recommend it. 